This item is the archived peer-reviewed author-version of:

\title{
Atomic structure of wurtzite CdSe (core)/CdS (giant shell) nanobullets related to epitaxy and growth
}

\section{Reference:}

Bladt Eva, van Dijk-Moes Relinde J. A., Peters Joep, Montanarella Federico, de Donega Celso Mello, Vanmaekelbergh Daniel, Bals Sara.- Atomic structure of wurtzite CdSe (core)/CdS (giant shell) nanobullets related to epitaxy and growth Journal of the American Chemical Society / American Chemical Society - ISSN 0002-7863 - 138:43(2016), p. 14288-14293 Full text (Publisher's DOI): http://dx.doi.org/doi:10.1021/JACS.6B06443

To cite this reference: http://hdl.handle.net/10067/1382510151162165141 


\title{
Atomic structure of wurtzite CdSe (core) / CdS (giant shell) nanobul- lets related to epitaxy and growth
}

\author{
Eva Bladt+, Relinde J. A. van Dijk - Moes*, Joep Peters*, Federico Montanarella*, Celso de Mello \\ Donega *, Daniël Vanmaekelbergh* and Sara Bals+ \\ + Electron Microscopy for Materials Research (EMAT), University of Antwerp, Groenenborgerlaan 171, B-2020 Antwerp, \\ $\mathrm{BE}$ \\ * Debye Institute for Nanomaterials Science, University of Utrecht, NL
}

KEYWORDS: CdSe/CdS Hetero-nanocrystals, Characterization techniques, Facet determination, Electron tomography

\begin{abstract}
Hetero-nanocrystals consisting of a CdSe core and a giant CdS shell have shown remarkable optical properties which are promising for applications in opto-electrical devices. Since these properties sensitively depend on the size and shape, a morphological characterization is of high interest. Here, we present a High Angle Annular Dark Field Scanning Transmission Electron Microscopy (HAADF-STEM) study of CdSe (core) / CdS (giant shell) hetero-nanocrystals. Electron tomography reveals that the nanocrystals have a bullet shape, either ending in a tip or a small dip, and that the CdSe core is positioned closer to the tip (or dip) than to the hexagonal base. Based on a high resolution HAADF-STEM study, we were able to determine all the surface facets. We present a heuristic model for the different growth stages of the CdS crystal around the CdSe core.
\end{abstract}

\section{INTRODUCTION}

Soon after the first successful synthesis of colloidal CdSe nanocrystals with reasonable photoluminescence quantum yield and size-dispersity ${ }^{1}$, it became clear that the growth of a shell of a higher band gap semiconductor, such as $\mathrm{ZnS}$ and $\mathrm{CdS}$, around the core increases the photoluminescence quantum yield and the photochemical stability ${ }^{2-6}$. This has, finally, resulted in synthetic routes for $\mathrm{CdSe} / \mathrm{X}$ core/shell systems with near-unit photoluminescence quantum yield, applicable as phosphors in LEDS, lasers, and as biological labels ${ }^{7-12}$. As a spin-off of this extensive research, it also became clear that the exciton wave functions and related optical properties could be engineered by the chemical composition and dimensions of the shell. In such a way, $\mathrm{CdSe} / \mathrm{X}$ hetero-nanocrystals were reported that (i) displayed a spatial separation of the electron and the hole wave functions ${ }^{13,14}$, (ii) reduced Auger recombination under high excitation densities ${ }^{15,16}$, (iii) reduced blinking when measured on the single quantum dot level ${ }^{17,18}$. This so-called wave function engineering demands a high control of the atomic epitaxy of the shell material on the CdSe core and of the growth of the shell material in case of thicker shells ${ }^{19-21}$. The characterization of $\mathrm{CdSe} / \mathrm{X}$ shell systems in real space with transmission electron microscopy (TEM) has been very helpful ${ }^{22}$ in this, but it should be realized that TEM is a projection method in which valuable information along one dimension is lost (i.e. the direction of the electron beam). For small $\mathrm{PbSe}$ (core) / CdSe (shell) hetero-nanocrystals, it has been shown that a three dimensional atomic characterization of the hetero-interface can be obtained by high angle annular dark- field scanning TEM (HAADF-STEM) tomography ${ }^{23}$. This was enabled by the relative small size of the $\mathrm{PbSe} / \mathrm{CdSe}$ heteronanocrystals and the large contrast between atomic columns containing $\mathrm{Pb}$ or $\mathrm{Cd}$. It is however far from straightforward to determine the three-dimensional atomic structure for much larger hetero-nanocrystals, e.g. consisting of a core and a giant shell as well as for structures of which the expected contrast between the two materials when imaging in HAADF-STEM mode is not strong.

CdSe (core) / CdS (giant shell) nanocrystals have a remarked scientific and technological importance ${ }^{16,21,24}$. It has been shown that giant $\mathrm{CdS}$ shell results in a enduring photochemical stability and a high photoluminescence quantum yield for excitons and bi-excitons, the latter due to strongly reduced Auger recombination ${ }^{16,21,25}$. This makes these systems of interest for high intensity applications in LEDs and lasers. It is clear that the optical properties depend on the shape and dimensions of the CdSe core and the CdS shell, which are on themselves determined by the crystal structure, i.e. zinc blende or wurtzite, and the conditions of the synthesis. The growth of a CdS shell around a zinc blende CdSe core leads to tetrapods, whereas in the case of wurtzite $\mathrm{CdSe}$ cores nanowires are formed, due to preferred growth in the [00-1] (c-axis) direc$\operatorname{tion}^{19}$. This demonstrates the importance of epitaxy of the CdS crystal on the facets of the CdSe structure.

Here, we present a three-dimensional atomic and chemical characterization of large $(30 \mathrm{~nm})$ hetero-nanocrystals, consisting of a $\mathrm{CdSe}$ core embedded in a giant shell of CdS grown by the successive ion layer adhesion and reaction (SILAR) meth- 
$\mathrm{od}^{26,27}$. We made use of a synthesis route to grow multiple CdS layers on wurtzite $\mathrm{CdSe}$ cores that leads to large nanocrystals with a bullet shape. The remarkable shape suggests that in this system the atomic epitaxy of CdS on CdSe and the peculiarities of $\mathrm{CdS}$ growth are important. In order to understand the initial growth, it is of crucial importance to locate the CdSe core in the bullet-shaped hetero-nanocrystal. In previous studies of $\mathrm{CdSe} / \mathrm{CdS}$ core/shell nanorods, the location of $\mathrm{CdSe}$ core could be retrieved from 2D TEM techniques such as strain analysis applied to high resolution transmission electron microscopy images ${ }^{28}$ and high resolution phase imaging ${ }^{29}$. Due to the large size and anisotropic shape of the bullets, 2D techniques can no longer be used and advanced electron tomography will be applied. Furthermore, the crystallography of the $\mathrm{CdS}$ growth is investigated by detailed imaging of the facets present at the surface of the bullet-shaped crystals. This characterization leads to a basic model for the formation of $\mathrm{CdSe}$ (core) / CdS (shell) nano-bullets.

\section{EXPERIMENTAL SECTION}

\section{Synthesis of CdSe dot in CdS bulk nanostructures}

The synthesis of the CdSe (core) / CdS (giant shell) heteronanocrystals (HNCs) was performed as follows: CdSe QD seeds were synthesized first ${ }^{20}$, and then a CdS multi-shell was grown onto CdSe QD seeds via the SILAR method ${ }^{10,20}$. In some cases, a final monolayer of $\mathrm{ZnS}$ was grown on top of the CdS; this last step had no effect on the shape of the HNCs. A more detailed description of the synthesis can be found in the supplementary information.

\section{Optical measurements}

Optical measurements were performed on diluted solutions of washed NCs in anhydrous toluene in quartz cuvettes. Absorption spectra were measured on a double-beam PerkinElmer Lambda $950 \mathrm{UV} /$ vis/NIR spectrophotometer. The photoluminescence spectra were recorded on an Edinburgh Instruments FLS920 spectrofluorometer equipped with a $450 \mathrm{~W}$ xenon lamp as excitation source and double grating monochromators. PL decay curves were acquired using an Edinburgh Instruments FLS920 spectrofluorometer and a pulsed diode laser (EPL-375 Edinburgh Instruments, 375 nm, 65 ps pulse width, $1 \mathrm{MHz}$ repetition rate) was used excitation source. Quantum Yield measurements were performed by reference to a dye, Lumogen red 305 (QY 95\%).

\section{X-ray and electron diffraction}

The low resolution TEM images (SI) and small area electron diffraction (SAED) images were performed on a FEI Tecnai-12 microscope operating at $120 \mathrm{kV}$. The SAED image is converted to graph which is typical for X-Ray Diffraction patterns. The angles used in this representation are calibrated with a $\mathrm{TlCl}$ reference sample. Samples were prepared by drop-casting a toluene solution of bullet particles onto a carbon-coated copper (400-Mesh) TEM grid.

\section{Transmission electron microscopy (TEM)}

Electron tomography experiments were performed using a FEI Tecnai G2 microscope operated at $200 \mathrm{kV}$. A Fischione tomography holder (model 2020) was used and tilt series were acquired in HAADF-STEM mode over an angular range of \pm $70^{\circ}$ with a tilt increment of $5^{\circ}$. The alignment of the series was performed using the Inspect 3D software.

High resolution HAADF-STEM images were acquired using a cubed FEI Titan microscope operating at $300 \mathrm{kV}$. A probe semiconvergence angle of $\sim 21 \mathrm{mrad}$ was used.

\section{RESULTS AND DISCUSSION}

Optical characterization of $\mathrm{CdSe} / \mathrm{CdS}$ core/shell HNCs. Figure S1 (Supporting Information) shows the absorption and emission spectra of the $\mathrm{CdSe} / \mathrm{CdS}$ core/shell heteronanocrystals in suspension. The extinction sets in at $800 \mathrm{~nm}$ and increases considerably with decreasing wavelength, to end with a clear feature at around $500 \mathrm{~nm}$. This feature is due to band edge absorption of the voluminous $\mathrm{CdS}$ shell. In most $\mathrm{CdSe}$ (core) / CdS (giant shell) systems, the CdS band edge absorption is so dominant that the optical transitions of the $\mathrm{CdSe}$ core are hardly visible in a linear plot of the absorptivity vs. wavelength. Here, a clear but featureless onset is observed at energies below the band gap of CdS, which can be assigned to light scattering. We remark that the $\mathrm{CdSe} / \mathrm{CdS}$ core/shell nanocrystals are relatively large colloidal particles, only stabilized by organic capping. As a consequence, small clusters of these bullets might be present in solution acting as light scattering centers. Nasilowski and coworkers attributed the increased absorption at energies below the bandgap of $\mathrm{CdS}$ and $\mathrm{CdSe}$ to partial alloying of Se and $\mathrm{S}$ in the CdSe core ${ }^{30}$. Our results, however, do not provide evidence for this. The luminescence spectrum is centered at around $663 \mathrm{~nm}$ with a FWHM of about $55 \mathrm{~nm}$, very much in agreement with the findings of Nasilowski et al. for the so-called graded CdSe (core) / CdS (giant shell) hetero-nanocrystals ${ }^{30}$.

The PL decay curve (Supporting Information, Figure S2) is far from single exponential. The average lifetime, defined as $\langle\tau\rangle=$ $\sum I_{i} t_{i} / \sum I_{i}$ with $I_{i}$ the intensity at delay time $t_{i}$ after the excitation pulse, decreases from $78.4 \mathrm{~ns}$ at $684 \mathrm{~nm}$ (the red side of the luminescence spectrum) to $71 \mathrm{~ns}$ at $640 \mathrm{~nm}$ (the blue side of the spectrum). These long life times suggest that they are dominated by radiative recombination of the exciton. We note that the decay rates are considerably slower than reported for type I CdSe $\mathrm{NCs}^{31,32}$.

These findings suggest a considerable delocalization of the electron part of the exciton wave function. Nasilowski et al. report that this leads to such a strong reduction of the Auger recombination that the bi-exciton PLQY can be as high as the exciton PLQY ${ }^{30}$. We remark that Vanmaekelbergh et al. has reported similar findings for $\mathrm{CdSe}$ core/CdS shells systems with thinner shells ${ }^{33}$. In line with the strong bi-exciton emission, Nasilowski et al. presents single dot emission traces with moderate jittering around a main emission intensity, without the presence of dark periods ${ }^{30}$; such emission traces have been coined "non-blinking" in the literature. We should remark here that the optical properties of $\mathrm{CdSe}$ (core) / CdS (giant shell) 
hetero-nanocrystals depend in a sensitive way on the epitaxy and atomic details ${ }^{30,34,35}$.

Morphology. Figure 1.a shows a HAADF-STEM overview image of the as synthesized $\mathrm{CdSe}$ (core) / CdS (giant shell) HNCs. It is clear that the $\mathrm{CdSe} / \mathrm{CdS}$ core/shell HNCs have an anisotropic shape, with hexagonal and bullet-like projections. In Figure 1.b two hexagonal projections of the HNCs are presented in more detail and an intensity difference in the center can be observed. This is further illustrated by the line profiles in Figure 1.c, $\mathrm{d}$ acquired along the white rectangles indicated in Figure 1.b. Two different types of profiles were observed: Type 1 shows a decrease of the intensity in the center, whereas a higher intensity is observed for type 2 . Since the intensity in HAADF-STEM images scales with sample thickness, the presence of a brighter region could be related to an increment in the projected thickness of the HNC. However, the intensity in HAADF-STEM also scales with the atomic number Z. Intensity changes may therefore also be related to the presence of the CdSe core. In order to elucidate the origin of the intensity changes, we applied electron tomography. Tilt series of HAADF-STEM images were acquired for each type of nanoparticle over a range of $\pm 70^{\circ}$.

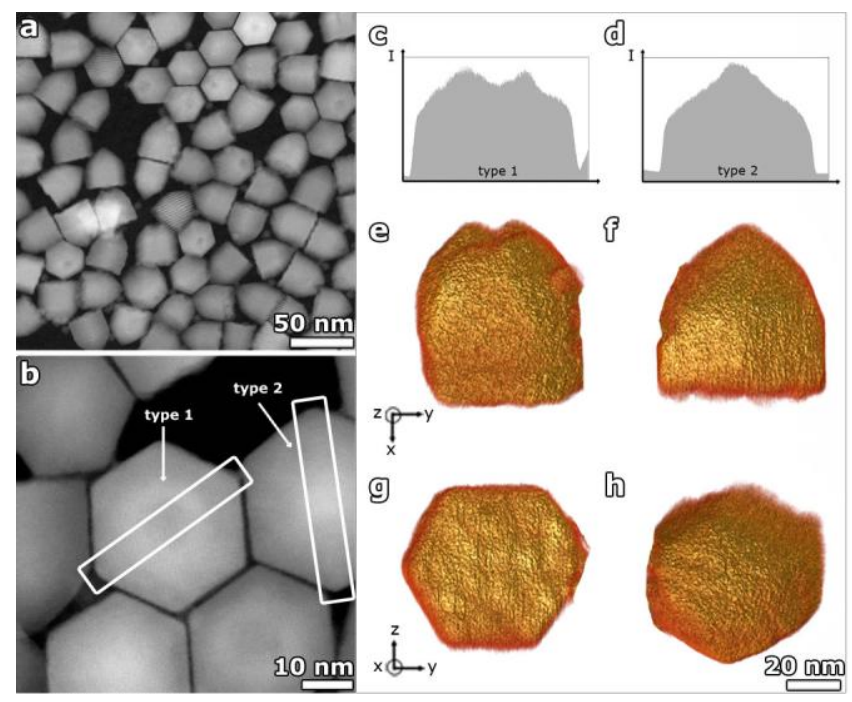

Figure 1. General shape of the CdSe (core) / CdS (giant shell) HNCs. (a) Overview HAADF-STEM image of the CdSe (core) / CdS (giant shell) HNCs demonstrating the bullet shape with a hexagonal basis. (b) HAADF-STEM image of several CdSe/CdS HNCs showing two types of intensity profiles: type 1 shows a lower intensity in the center of the structure, type 2 a higher intensity. (c,d) Intensity profiles acquired along the white rectangles in (b) are presented, suggesting bullets with a dip or a tip on one end. (e-h) 3D reconstructions unambiguously demonstrate the two different bullet-type crystal shapes; both have a hexagonal base $(\mathrm{g}, \mathrm{h})$, however, type 1 ends in a dip (e) and type 2 in a tip (f).

In Figure 1.e-h, the 3D reconstructions are presented, showing two different bullet-type crystal shapes: both have a hexagonal base (Figure 1.g and 1.h), but at the other end either a dip (Figure 1.e) or a tip (Figure 1.f) is observed. This explains the intensity differences in the center of the HNCs observed in the
2D HAADF-STEM images. It must be noted that 2D overview images enabled us to estimate that both types of crystal shape are roughly equally present in the samples that we investigated.

Core location. Previous work on $\mathrm{CdSe} / \mathrm{CdS}$ core/shell nanorods showed that the CdSe core can be located from 2D high resolution real-space or phase images ${ }^{29,36}$. Recently, X-ray energy dispersive spectroscopy (XEDS) has been applied to visualize the $\mathrm{CdSe}$ core in a $\mathrm{CdSe} / \mathrm{CdS}$ quantum dot in a $2 \mathrm{D}$ projection $^{35}$. Because of the larger size of the crystals and the bullet shape, a 3D characterization is indispensable in the present case. Despite of the small difference in atomic number $\mathrm{Z}$ between the core and the shell, HAADF-STEM tomography is the preferred technique to investigate the location of the core. Although the use of chemical mapping such as electron energy loss spectroscopy (EELS) or XEDS might be better suited to identify the CdSe core in the HNCs, applying these techniques in $3 \mathrm{D}$ was not feasible since the required electron dose induced severe beam damage.

A HAADF-STEM series was acquired over a tilt range of \pm $70^{\circ}$ with a tilt increment of $5^{\circ}$. When inspecting orthoslices through the $3 \mathrm{D}$ reconstruction, computed using a conventional algorithm (SIRT), the CdSe core is faintly visible, therefore a correct detection of the position is difficult (Supporting Information, Figure S4). In order to better visualize the CdSe core $\left(Z_{\mathrm{Se}}=34\right)$ in the $\mathrm{CdS}$ shell $\left(\mathrm{Z}_{\mathrm{S}}=16\right)$, we applied a more advanced reconstruction algorithm. The so-called total variation minimization reconstruction algorithm ${ }^{37}$ (TVM) is based on the assumption that the gradient of the object under investigation is sparse. As a consequence, edges in the 3D reconstruction will be enhanced. Orthoslices, acquired at the same position as Figure S4 (Supporting Information) are presented in Figure 2.c,f. The results show that the core is not located in the center of the CdS crystal (Fig 2.a,b and 2d,e), but closer to the tip or dip of the bullets. Previous studies on $\mathrm{CdSe} / \mathrm{CdS}$ core/shell nanorods also showed an asymmetric position of the $\mathrm{CdSe}$ core along the nanorod axis ${ }^{19,28,29,38}$. Overall, the distance between the $\mathrm{CdSe}$ core position and the hexagonal base is comparable for both bullet shapes, suggesting a very similar growth process for both morphologies.

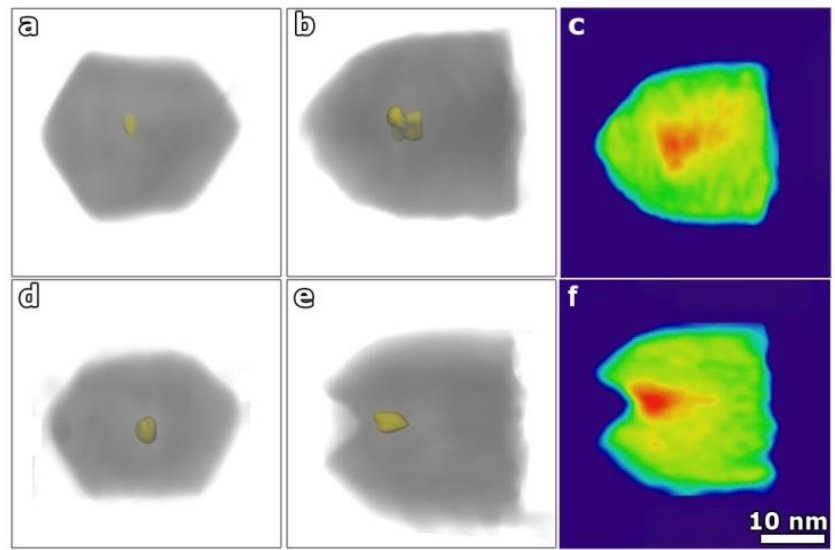

Figure 2. HAADF-STEM tomography on bullet-shaped $\mathrm{CdSe} / \mathrm{CdS}$ hetero-nanocrystals to detect the position of the $\mathrm{CdSe}$ core. The location of the CdSe core is shown in yellow in the $3 \mathrm{D}$ reconstructions of the two types of bullet-shaped 
HNCs, type 2 with a tip (a,b) and type 1 with a dip (d,e). When applying the total variation minimization reconstruction algorithm, the heavier CdSe core is clearly visible in the orthoslices (c and f).

Facet Determination. As the two types of bullet-shaped nanocrystals yield a similar position for the CdSe core, the epitaxy and growth of the CdS crystalline shell is expected to be very similar for both types. In order to evaluate the surface energy of both types, the facets of the HNCs need to be identified. Previous studies have shown that the facets of nanocrystals can be investigated by electron tomography ${ }^{39-41}$. Here, this is not straightforward because of the relatively small number of projection images in the tilt series. The lack of information will induce a more rounded shape, which hampers an unambiguous characterization of the facets from a 3D experiment. Therefore, we additionally used direct high resolution HAADF-STEM imaging to determine the facets of the $\mathrm{CdSe} / \mathrm{CdS}$ bullets. Figure 3 shows a high resolution HAADFSTEM image acquired along the (long) [001] direction. The hexagonal arrangement of the atomic columns (Figure 3.c) at the base of the HNC demonstrates that the $\mathrm{CdSe} / \mathrm{CdS}$ core/shell HNCs have a wurzite crystal structure. Since the wurzite structure is non-centrosymmetric, anisotropic growth is expected, which is confirmed by the position of the CdSe core (see above). However, this is in disagreement with the principles of the SILAR method designed for layer-by-layer growth. It is well possible, that even in the SILAR method, the growth occurs by molecular attachment of CdS units instead of $\mathrm{Cd}$ and $\mathrm{S}$ in subsequent steps ${ }^{42-44}$. This would preserve the facet polarity and anisotropy. Moreover, a slight excess of the added precursors would enable preferential growth in the polar [00-1], S-terminated direction. Next to the identification of the crystal system, the lateral facets can be examined from the high resolution STEM image (Figure 3.a) and its corresponding diffractogram (Figure 3.b). From these images, the lateral facets are identified as $\{-110\}$ planes. In Figure 3.d, a schematic overview is shown to support the viewing directions with respect to the hexagonal base of a HNC.

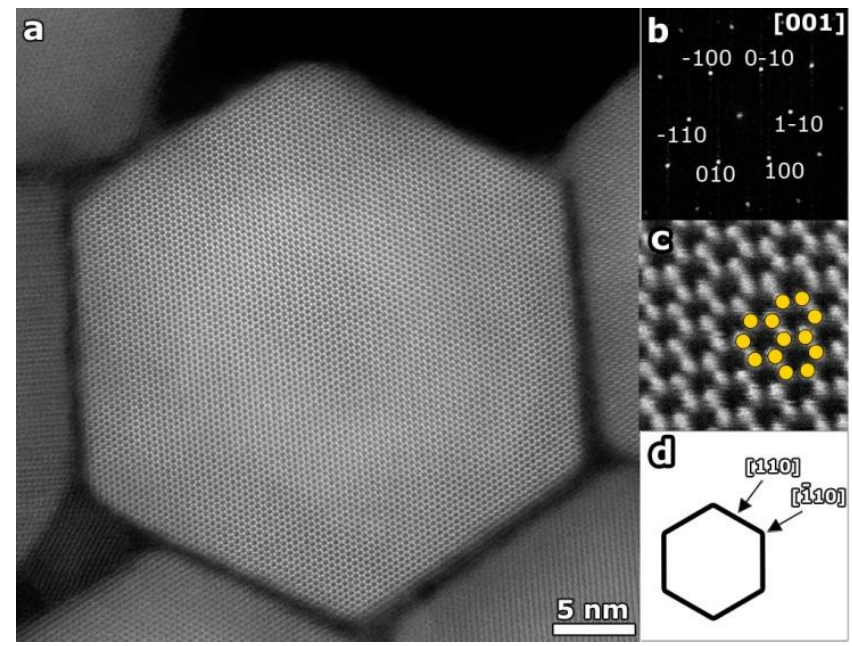

Figure 3. Atomically resolved HAADF-STEM image acquired along the long axis of the bullets. (a) High resolution HAADFSTEM image of a CdSe/CdS bullet-shaped nanocrystal with its corresponding diffractogram (b). The image acquired along the [001] direction shows the hexagonal pattern characteristic for wurtzite. (c) A more detailed view of the atomic arrangement is shown. (d) Schematic of the base of a CdSe/CdS HNC with indication of the [110] and [-110] viewing directions.

In order to investigate the facets that form the tip or the dip, high resolution images acquired along an edge between two lateral facets, corresponding to the [110] direction, are evaluated. In this direction, the angle between a lateral facet and a facet forming the tip can be measured. In this manner, we conclude that the facets at the tip correspond to $\{1-11\}$ planes. For the type $1 \mathrm{CdSe} / \mathrm{CdS}$ bullets with a dip, the $\mathrm{d} 1$ and d4 facets (Figure 4.c) are identical to the facets forming the tip of the type 2 bullets. The dip forming facets were identified by studying the decrease in intensity in the high resolution HAADF-STEM images due to the presence of the dip. A more detailed comparison is shown in Figure 4.b and 4.d. Analyzing the $\mathrm{d} 2$ and $\mathrm{d} 3$ facets in Figure 4.c shows that the $\mathrm{d} 2$ facet is parallel to the $\mathrm{d} 4$ facet and $\mathrm{d} 3$ is parallel to $\mathrm{d} 1$. This shows that both types of bullet shapes have identical facets, and a comparable total surface energy.

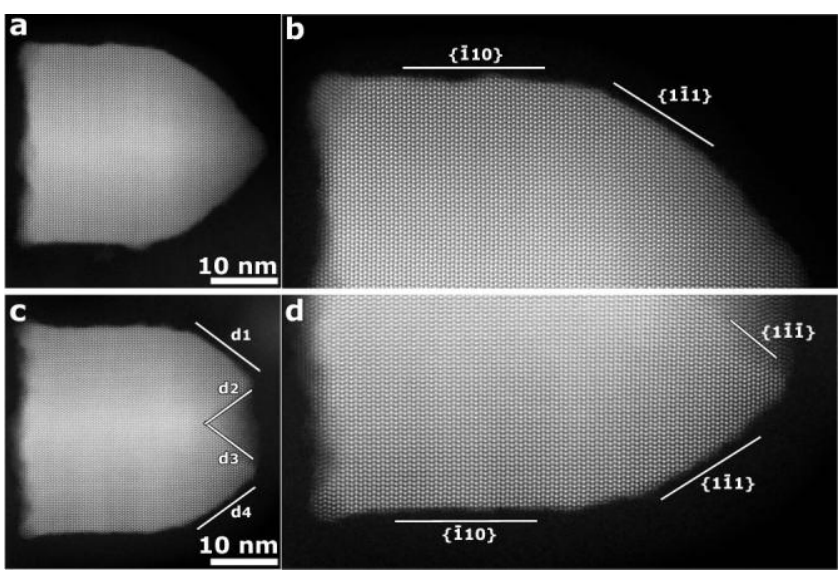

Figure 4. Identification of the facets at the tip or dip of the $\mathrm{CdSe} / \mathrm{CdS}$ bullet-shaped HNCs. (a,c) High resolution HAADF-STEM images of both types of bullet-shaped nanocrystals, with a more detailed view in $(b, d)$. The la-teral facets correspond to [-110] planes. For both types of bullet shapes, the facets originating from the lateral facets towards the dip or tip correspond to [1-11] facets. The type 1 HNCs ending in a dip have additional facets ending in the dip, indicated as ( $d 2$ and d3). In (d) it is shown that these facets (d2, d3) are parallel to facet $\mathrm{d} 1$ and $\mathrm{d} 4$, respectively.

The complete characterization of the atomic structure and facets of the bullet-shaped CdSe (core) / CdS (giant shell) HNCs suggest a mechanism of CdS epitaxy and growth. It was mentioned above that the growth most probably occurs by attachment of CdS units, rather than separate Cd and S layers. The presence of excess CdS molecular units allows a distinct faster growth of the S-terminated [00-1] facets in the direction of the polar c-axis ${ }^{43}$. An atomic simulation of the growth of the giant $\mathrm{CdS}$ crystal around the CdSe core is beyond the scope of the present work. Here, we present an intuitive picture, based on 
the atomic characterization of the bullet-shaped heteronanocrystals present above, schematized in Figure 5.

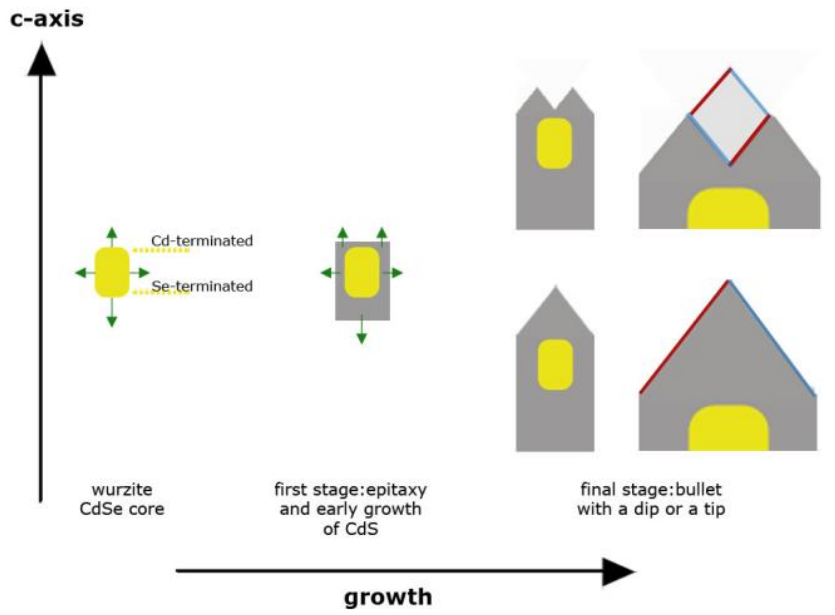

Figure 5. Scheme of the anisotropic growth of the giant $\mathrm{CdS}$ shell on the CdSe core. From left to right: slightly elongated wurtzite $\mathrm{CdSe}$ crystal core; the green arrows indicate the relative growth rates of the $\mathrm{CdS}$ in the [001], [00-1] and [110] directions. In the first stage (Figure S5), there is epitaxy and starting growth of a hexagon-based CdS crystal. In later stages show either a growth in the [001] on top of the crystal accompanied by horizontal ingrowth, resulting in a bullet with a dip; or a growth of the inward facets which result in bullets with a tip. The facets forming the dip are indicated in red and blue to show that a bullet with a tip is build up by exactly the same facets.

The CdSe core has a wurtzite crystal structure, and in-duces wurtzite growth of the CdS shell (Supporting Information, Figure S5). In the early stage of the process, epitaxial formation of CdS occurs on all facets, but the growth of wurtzite $\mathrm{CdS}$ in the [00-1] direction with a S-terminated facet is faster than in the opposite [001] direction terminated by $\mathrm{Cd}$. This is similar to the growth of $\mathrm{CdSe} / \mathrm{CdS}$ rods. Such strongly anisotropic growth rates are well documented for wurtzite type crystals $^{45-47}$. In a later stage, the cylindrical crystal shape with hexagonal base typical for wurtzite crystals becomes increasingly pronounced. The growth along the [00-1] is fast with respect to the opposite direction and the 6 lateral directions of the CdS crystal, which grow more slowly. Growth in the direction perpendicular to the c-axis can also occur inwards (arrows not shown) resulting in a bullet shape with a dip. Possibly, the dip width and depth is gradually reduced by inwards $\mathrm{CdS}$ growth. In any case, stable [1-11] facets present in the dip lead to a low surface energy, and slow growth. It remains ambiguous whether the type 1 and type 2 bullet-shaped heteronanocrystals are equally stable polymorphs, or alternatively, that the type 1 bullets are an unfinished stage, finally resulting into the final bullet-shaped HNCs with a tip. However, based on the facet characterization and equal presence of both types of morphologies, it is suggested that both types are equally stable. The end-facets at the tip indicate that the $\{1-11\}$ facets are more stable than the Cd-terminated $\{001\}$ facets.

\section{CONCLUSIONS}


M. G. Nat. Mater. 2013, 12, 445 .

Piryatinski, A.; Ivanov, S. A.; Tretiak, S.; Klimov, V. I. Nano Lett. 2007, 7, 108.

(14) Allione, M.; Ballester, A.; Li, H.; Comin, A.; Movilla, J. L.; Climente, J. I.; Manna, L.; Moreels, I. ACS Nano 2013, 7, 2443.

(15) Mangum, B. D.; Wang, F.; Dennis, A. M.; Gao, Y.; Ma, X.; Hollingsworth, J. A.; Htoon, H. Small 2014, 10, 2892.

(16) Htoon, H.; Malko, A. V.; Bussian, D.; Vela, J.; Chen, Y.; Hollingsworth, J. A.; Klimov, V. I. Nano Lett. 2010, 10, 2401.

(17) Malko, A. V.; Park, Y.-S.; Sampat, S.; Galland, C.; Vela, J.; Chen, Y.; Hollingsworth, J. A.; Klimov, V. I.; Htoon, H. Nano Lett. 2011, 11, 5213.

(18) Zhao, J.; Chen, O.; Strasfeld, D. B.; Bawendi, M. G. Nano Lett. 2012, 12, 4477.

(19) Talapin, D. V.; Nelson, J. H.; Shevchenko, E. V.; Aloni, S.; Sadtler, B.; Alivisatos, A. P. Nano Lett. 2007, 7, 2951.

(20) Greytak, A. B.; Allen, P. M.; Liu, W.; Zhao, J.; Young, E. R.; Popović, Z.; Walker, B.; Nocera, D. G.; Bawendi, M. G. Chem. Sci. 2012, 3, 2028.

(21) Christodoulou, S.; Vaccaro, G.; Pinchetti, V.; De Donato, F.; Grim, J. Q.; Casu, A.; Genovese, A.; Vicidomini, G.; Diaspro, A.; Brovelli, S.; Manna, L.; Moreels, I. J. Mater. Chem. C 2014 2, 3439 .

(22) van Embden, J.; Jasieniak, J.; Mulvaney, P. J. Am. Chem. Soc. 2009, 131, 14299.

(23) Bals, S.; Casavola, M.; van Huis, M. A.; Van Aert, S.; Batenburg, K. J.; Van Tendeloo, G.; Vanmaekelbergh, D. Nano Lett. 2011, 11, 3420.

(24) Mangum, B. D.; Sampat, S.; Ghosh, Y.; Hollingsworth, J. a; Htoon, H.; Malko, A. V. Nanoscale 2014, 6, 3712.

Pal, B. N.; Ghosh, Y.; Brovelli, S.; Laocharoensuk, R.; Klimov, V. I.; Hollingsworth, J. A.; Htoon, H. Nano Lett. 2012, 12, 331.

Qu, L.; Peng, Z. A.; Peng, X. Nano Lett. 2001, 1, 333.

Li, J. J.; Wang, Y. A.; Guo, W.; Keay, J. C.; Mishima, T. D.; Johnson, M. B.; Peng, X. J. Am. Chem. Soc. 2003, 125, 12567.

Carbone, L.; Nobile, C.; De Giorgi, M.; Sala, F. Della; Morello, G.; Pompa, P.; Hytch, M.; Snoeck, E.; Fiore, A.; Franchini, I. R.; Nadasan, M.; Silvestre, A. F.; Chiodo, L.; Kudera, S.; Cingolani, R.; Krahne, R.; Manna, L. Nano Lett. 2007, 7, 2942.

McBride, J.; Treadway, J.; Feldman, L. C.; Pennycook, S. J.; Rosenthal, S. J. Nano Lett. 2006, 6, 1496.

Nasilowski, M.; Spinicelli, P.; Patriarche, G.; Dubertret, B Nano Lett. 2015, 15, 3953.

van Driel, A. F.; Allan, G.; Delerue, C.; Lodahl, P.; Vos, W. L.; Vanmaekelbergh, D. Phys. Rev. Lett. 2005, 95, 236804.

de Mello Donegá, C.; Koole, R. J. Phys. Chem. C 2009, 113 , 6511.

Vanmaekelbergh, D.; van Vugt, L. K.; Bakker, H. E.; Rabouw, F. T.; Nijs, B. De; van Dijk-Moes, R. J. A.; van Huis, M. A.; Baesjou, P. J.; van Blaaderen, A. ACS Nano 2015, 9, 3942.

Ghosh, Y.; Mangum, B. D.; Casson, J. L.; Williams, D. J.; Htoon, H.; Hollingsworth, J. A. J. Am. Chem. Soc. 2012, 134, 9634.

Orfield, N. J.; McBride, J. R.; Wang, F.; Buck, M. R.; Keene, J. D.; Reid, K. R.; Htoon, H.; Hollingsworth, J. A.; Rosenthal, S. J. ACS Nano 2016, 10, 1960.

Bertoni, G.; Grillo, V.; Brescia, R.; Ke, X.; Bals, S.; Catellani, A.; Li, H.; Manna, L. ACS Nano 2012, 6, 6453.

Goris, B.; Roelandts, T.; Batenburg, K. J.; Heidari Mezerji, H.; Bals, S. Ultramicroscopy 2013, 127, 40.

van der Stam, W.; Bladt, E.; Rabouw, F. T.; Bals, S.; de Mello Donega, C. ACS Nano 2015, 9, 11430.

Weyland, M.; Yates, T. J. V.; Dunin-Borkowski, R. E.; Laffont, L.; Midgley, P. A. Scr. Mater. 2006, 55, 29.

González, J. C.; Hernández, J. C.; López-Haro, M.; del Río, E.;
Midgley, P. A.; Calvino, J. J. Angew. Chemie 2009, 121, 5417.

Goris, B.; Bals, S.; Van den Broek, W.; Carbó-Argibay, E.; Gómez-Graña, S.; Liz-Marzán, L. M.; Van Tendeloo, G. Nat. Mater. 2012, 11, 930. 129, 305 .

de Mello Donegá, C. Chem. Soc. Rev. 2011, 40, 1512.

Abe, S.; Čapek, R. K.; De Geyter, B.; Hens, Z. ACS Nano 2012 , 6,42 .

Manna, L.; Milliron, D. J.; Meisel, A.; Scher, E. C.; Alivisatos, A. P. Nat. Mater. 2003, 2, 382.

Manna, L.; Wang; Cingolani, R.; Alivisatos, A. P. J. Phys. Chem. B 2005, 109, 6183.

Moore, D.; Wang, Z. L. J. Mater. Chem. 2006, 16, 3898. Delgado, J. J.; Hungría, A. B.; Trasobares, S.; Bernal, S.; 


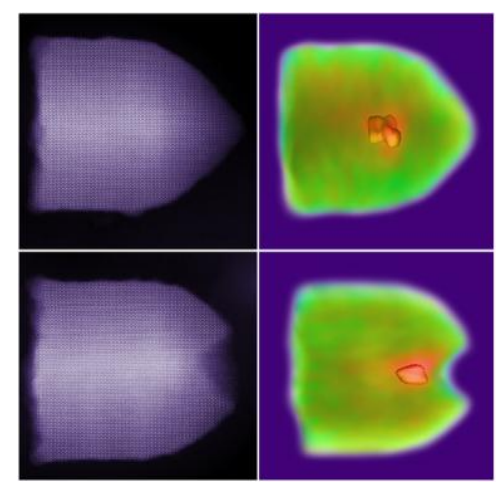

